\title{
Research on a Localization Algorithm Based on Wireless Sensor Network
}

\author{
Xuelong Zhang ${ }^{1}$ and Hao Liu ${ }^{2, *}$ \\ ${ }^{1}$ Changsha Normal University, Changsha 4101000, China \\ ${ }^{2}$ Hunan University of Humanities, Science and Technology, \\ Hunan 417000, China \\ 1365169810@qq.com and 2hhd0407@126.com \\ 2, *Corresponding author
}

\begin{abstract}
After analyzing the disadvantages of the centralized multidimensional localization algorithms MDS-MAP (Multi-Dimensional Scaling-MAP) in positioning accuracy and computational complexity, we present a new localization algorithm based on a set of statistical vectors (SV). The solving equation of the double centered matrix can be simplified by node coordinate transformation. In order to reduce the noise disturbance and decrease the effect of ranging error on the followed location accuracy, a new coordinate inner product matrix can be reconstructed by using a set of statistical vectors, which can be used to calculate the node coordinates directly. This algorithm can realize centralized localization, distributed localization and incremental localization of nodes.
\end{abstract}

Keywords: wireless sensor network, node, localization algorithm

\section{Introduction}

During the WSNs positioning, generally it assumes neighboring nodes can communicate with one another and the distance among them can be surveyed. Basically, the smaller the measured distance error, the higher the positioning precision can achieve [1-2]. So it's hoped that the distance measurement error can be decreased by modifying node's hardware structure or increasing node's transmitting power [3-4]. However, high precision distance measuring means will bring about increased hardware cost and energy consumption. For instance, the distance measurement technique TOA requires strict time synchronization; TDOA requires two signal transmitting devices; AOA needs multiple signal receiving devices. Increasing node's transmitting power will shorten the shelf life of wireless sensor nodes. All those go against the design principle of low cost and low consumption of WSNs [5-7].

Among the four distances ranging techniques like RSSI, TOA, TDOA and AOA, the positioning system based on RSSI is of the lowest hardware cost and can be easily implemented [8]. That's why it's favored the most by scholars' home and abroad. But comparatively, it causes the biggest distance measuring error. RSSI's basic principle is: to convert the attenuation of signal strength to signal propagation distance; with extending of transmitting distance, the signal intensity of wireless signal would decay according to certain rules. Yet, the propagation feature of signal is related with environmental parameters; signal damping is affected by many factors such as temperature, humidity, obstacles, propagation mode and multipath effect [9-10]. Hence it's quite difficult to establish a uniform signal transmit attenuation model. Besides, the height of radio frequency antenna is another important factor affecting signal transmit attenuation model. The precision of positioning algorithm based on RSSI is associated not only with the distance measuring accuracy and also with features of the positioning algorithm itself. In academic circles, lots of positioning algorithms based on RSSI were proposed, e.g. well- 
known RADAR positioning system, multiple point distance measurement positioning method, maximum posterior probability position technique based on model and map [11].

The paper is designed to discuss positioning problem based on RSSI distance measurement. In order to reduce the influence of distance measuring error on positioning precision, it proposed the positioning algorithm based on statistical vector set (SV). Through coordinate conversion, the method simplifies the solution equation for centralized coordinate matrix and utilizes statistically uncorrelated vector set to reconstruct coordinate inner product matrix, reducing the noisy disturbance in range measurement. With direct use of coordinate inner product matrix, the coordinate of node can be calculated. Experiment shows that, in the case of big distance measuring error, SV positioning algorithm can effectively improve positioning precision, rather suitable for node positioning based on WSNs with lower cost hardware.

Suppose communication distance among all nodes is the same in the network; nodes between two can communicate through one or more hops; also each node can send and receive signal and has function of calculating signal strength. In the paper, CC2430 of Texas Instrument is used as main chip of sensor node as to receive signal. In order to validate the practicability of the proposed algorithm, considering the error of RSSI signal would be bigger, here in the simulation part, we use Noisy Disks model to emulate any possible distance measuring error caused by RSSI in the way of directly increasing distance measuring error.

\section{SV Positioning Algorithm}

\subsection{Centralized SV Positioning Algorithm}

Centralized positioning algorithm is the kind of technique which concentrates all required information to one central node of computer then performs positioning of single or all nodes from there. Suppose original coordinate of $\mathrm{N}$ nodes $\left\{X_{1}, X_{2}, \ldots, X_{N}\right\}$ is $x_{i}^{\prime}\left(x_{i}^{\prime} \in R^{d}\right) ; N=\{1,2, \ldots, N\}$ means the collection of sensor nodes. Translate all nodes to make $X_{1}^{\prime}$ at original point of coordinate and the translation amount is $a=\left(a_{1}, a_{2}, \ldots, a_{d}\right)$; then define new coordinate as $x_{i}^{\prime}\left(x_{i}^{\prime} \in R^{d}\right)$, where $X_{1}^{\prime}$ can be any node of referential nodes; then after translation, the coordinate matrix of referential node is $X_{N}=\left\{X_{1}, X_{2}, \ldots, X_{N}\right\}^{T}$. The two-two distances among all nodes constitute measuring matrix $D_{N}=\left(\mathscr{d}_{i j}^{o}\right)_{M \times N}$; then measuring distance among nodes is:

$$
\mathscr{g}_{i j}^{o}=N\left(d\left(x_{i}, x_{j}\right), \sigma\right)
$$

$d\left(x_{i}, x_{j}\right)$ is the Euclidean distance between $X_{i}$ and $X_{j}$ for the sensor nodes. In order to get the coordinates of the nodes between the inner product, the introduction of coordinate inner product matrix $B_{N}=\left(b_{i j}\right)_{N \times N} . b_{i j}$ is:

$$
b_{i j}=\left(\mathscr{d}_{i 1}^{\mathbb{E}}+\mathscr{d}_{1 j}^{\bar{E}}-\mathscr{d}_{i j}^{\mathbb{E}}\right) / 2
$$

Since measured value $\mathscr{d}_{i j}^{o}$ has error, the correlation of data which are interrelated at $\mathrm{d}$ dimension is forced to extend towards high dimension and decompose characteristic value of $B_{N}$; the number of characteristic value which is bigger than 0 will be more than d; if the first $\mathrm{d}$ feature values and relevant feature vector are chosen to form according coordinates, the error will be bigger. 
In WSNs, since $B_{N}$ is usually singular, there is at least one characteristic value with 0 as to do characteristic decomposition of $B_{N}$, which will cause nonexistence of inverse matrix of feature value matrix. The characteristic decomposition of $B_{N}$ is expressed as below:

$$
B_{N}=\left(V_{1}, V_{2}\right)\left(\begin{array}{ll}
D_{1} & 0 \\
0 & D_{2}
\end{array}\right)\left(\begin{array}{l}
V_{1}^{T} \\
V_{2}^{T}
\end{array}\right)
$$

The coordinates of unknown nodes can be calculated by $X_{N} x_{k}^{T}=b_{k}^{\prime}$ :

$$
x_{k}^{T}=\left(X_{N}^{T} X_{N}\right)^{-1} X_{N}^{T} b_{k}^{\prime}
$$

To sum up the derivation process, the centralized SV location algorithm includes the following steps:

Step 1: translation of the reference node, so that a reference node is located in the origin of the coordinates;

Step 2: according to the signal strength nodes, calculate the distance two points;

Step 3: use the formula (3) to calculate the coordinates of the inner product matrix;

Step 4: to coordinate the inner product matrix center matrix decomposition, take more than zero eigenvalues of the coordinates of the inner product matrix feature vector is constructed and the corresponding;

Step 5: use the formula (4) to calculate the unknown node coordinates, and translated into the original coordinate system.

\subsection{Distributed SV Positioning Algorithm}

The distributed positioning algorithm needs to communicate only with neighboring nodes during positioning. The positioning process is completed within the nodes. In the distributed SV positioning algorithm, it uses only the coordinate of anchor nodes and the distance information between specified unknown nodes and anchor nodes to realize distributed positioning of the specified unknown nodes.

Suppose in WSNs, there are $\mathrm{K}$ anchor nodes like $\left\{X_{1}, X_{2}, \ldots, X_{k}\right\}$, and original coordinate is $x_{i}^{\prime}\left(x_{i}^{\prime} \in R^{d}\right) ; K=\{1,2, \ldots, K\}$ means the collection of $\mathrm{K}$ anchor nodes. Do coordinate translation of all anchor nodes to make $X_{1}^{\prime}$ at original point of anchor and translation amount is $a=\left(a_{1}, a_{2}, \ldots, a_{d}\right)$; new coordinate is defined as $x_{i}^{\prime}\left(x_{i}^{\prime} \in R^{d}\right)$, where $X_{1}^{\prime}$ can be any of anchor nodes; then after translation, the coordinate matrix of anchor nodes is $X_{N}=\left\{X_{1}, X_{2}, \ldots, X_{k}\right\}^{T}$. Since coordinate information of anchor nodes is known, so the distance among them is determined. The two-two distance among those anchor nodes form measuring matrix $D_{K}=\left(d_{i j}\right)_{K \times K} \cdot d_{i j}$ is the Euclidean distance between nodes.

Similarly, In order to get product relationship between node coordinates, the coordinate inner product matrix $B_{K}=\left(b_{i j}\right)_{K \times K}$ is introduced. $b_{i j}$ is:

$$
b_{i j}=\left(d_{i 1}^{2}+d_{1 j}^{2}-d_{i j}^{2}\right) / 2
$$

In the distributed positioning system, unknown nodes acquire parameter $\alpha, D_{K}$ and $B_{K}$ in these two means:

(1) store all the three parameters in unknown nodes;

(2) send to unknown nodes via wireless signal. 
In WSNs, for any unknown node $\mathrm{K}+1$, the ranging distance $\mathscr{d}_{1,(k+1)}^{0}, \mathscr{d}_{2,(k+1)}^{0}, \ldots, \mathscr{d}_{k,(k+1)}^{o}$ of it between all other anchor nodes can be obtained by measuring technique. We assume unknown nodes can communicate with all anchor nodes.

Because of $b_{i j}=b_{j i}$, so $B_{K+1}$ can coordinate the inner product matrix represented by the formula:

$$
B_{K+1}=\left[\begin{array}{cc} 
& \\
B_{k} \ldots \ldots \ldots . . & \\
& b_{1,(k+1)} \\
b_{1,(k+1)} \ldots b_{k,(k+1)} & b_{(k+1),(k+1)}
\end{array}\right]
$$

Like the centralized SV location algorithm, $B_{K+1}$ is usually singular, there is at least one characteristic value of 0 when $B_{K+1}$ is decomposed, this will result in the inverse matrix of the eigenvalue matrix not exist. Eigenvalue decomposition of $B_{N}$ :

$$
B_{k+1}=\left(V_{1}, V_{2}\right)\left(\begin{array}{ll}
D_{1} & 0 \\
0 & D_{2}
\end{array}\right)\left(\begin{array}{l}
V_{1}^{T} \\
V_{2}^{T}
\end{array}\right)
$$

To sum up the derivation process, the distributed SV location algorithm includes the following steps:

Step 1: translation of the reference node, so that a reference node is located in the origin of the coordinates;

Step 2: according to the actual coordinates of the anchor node, the distance between the anchor node is calculated, and the anchor node matrix $D_{K}, B_{K}$;

Step 3: the distance between the unknown node $\mathrm{K}+1$ and all other anchor nodes is obtained by ranging technology $g_{1,(k+1)}^{0}, g_{2,(k+1)}^{o}, \ldots, d_{k,(k+1)}^{0}$.Using equation (6) generate the coordinates of the inner product matrix $B_{k+1}$;

Step 4: to coordinate the inner product matrix $B_{k+1}$ of feature value decomposition and take greater than zero in the characteristic value and the corresponding eigenvectors of the new coordinate inner product matrix;

Step 5: the formula (7) is used to calculate the coordinates of the unknown node $\mathrm{K}+1$, and the translation to the original coordinate system.

\section{Experiment Design and Discussion}

In order to verify the performance of SV positioning algorithm, we need firstly test the proposed algorithm with RSSI based measuring technique; then use Noisy Disk model to simulate the performance of SV positioning algorithm in many cases of distance measuring error.

\subsection{Experimental Platform and Positioning Result}

The distance measurement technique based on RSSI can get the distance from signal point to receiving point with signal propagation attenuation model according to the strength or weakness of received RSSI signal. Since RSSI is susceptible to environment, there is no fixed propagation error model; instead, it needs to determine model parameters after on-site survey as per actual environment.

Here we choose CC2430 as the main chip to make experimental nodes. CC2430 is the first $2.4 \mathrm{GHz}$ RF system single chip which complies with ZigBee technology. It still uses the infrastructure of CC2420 and integrates ZigBee RF front end, memory and micro- 
controller into a single chip. The current loss during its working is $27 \mathrm{~mA}$, which is respectively lower than $27 \mathrm{~mA}$ or $25 \mathrm{~mA}$ in receiving or transmitting pattern. Its hardware supports CSMA/CA function and digitized RSSI/LQI and strong DMA function. Each node can receive/send RF signal. When one node sends signal, the other nodes can receive it and surveys its RSSI. Here under we estimate parameters of RSSI signal transmit error model in the laboratory.

3.1.1. RSSI signal transmit attenuation model [12]: Define $P_{i j}$ as the strength $(\mathrm{mW})$ of signal which is sent by node $\mathrm{j}$ and received by node $\mathrm{i}$; and assume $P_{i j}=P_{j i} ; P_{i j}$ is random variable of logarithmic normal distribution; $P_{i j}(\mathrm{dBm})=10 \log _{10} P_{i j}$ complies with Gaussian distribution [13-14].

$$
\begin{gathered}
P_{i j}(d B m): N\left(P_{i j}(d B m), \sigma_{d B}^{2}\right) \\
\bar{P}_{i j}(d B m)=P_{0}(d B m)-10 n_{p} \log _{10}\left(d_{i j} / d_{0}\right)
\end{gathered}
$$

Used CC2430 to make nodes to send and receive RF signal; measure RSSI value to confirm the statistics model mentioned above. In order to get the above model, it requires to determine three parameters: $d_{0}, P_{0}(d B m)$ and $n_{p}$. Usually $d_{0}=1 m ; P_{0}(d B m)$ is measured RSSI value when the distance $d_{i j}=1 \mathrm{~m}$ between node $X_{1}$ and $X_{2}$. When $d_{0}$ and $P_{0}(\mathrm{dBm})$ are both determined, put node $X_{1}$ and $X_{2}$ in different places; then try several times to get RSSI value of them and substitute to equation (9); after calculation of a few times, the mean value of $n_{p}$ is solved. Till this moment, three parameters of RSSI signal transmit attenuation model are all obtained.

The red circle in Figure 1 is the average value of the RSSI signal measured at different distances from the sensor nodes. In this experiment, each position is measured 10 RSSI values, and then the average. According to the measured value, the parameters of RSSI signal transmission attenuation model can be $P_{0}(\mathrm{dBm}) \approx-45, n_{p} \approx 3$. It is shown in Figure1.

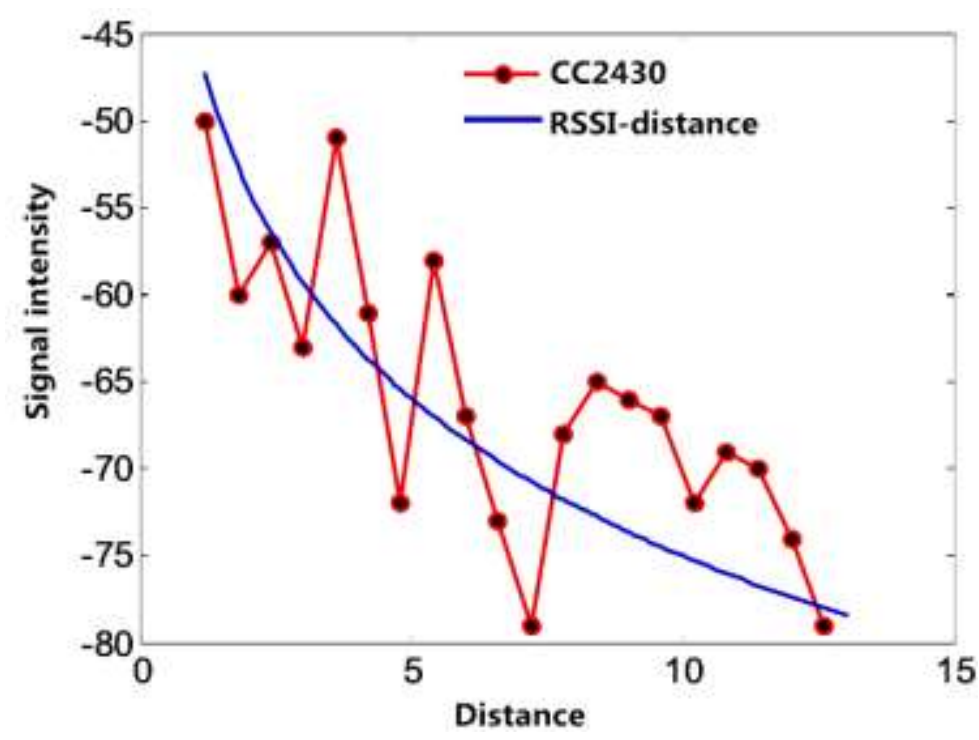

Figure 1. Relationship between RSSI and Distance

Experiment finds that for two fixed sensor nodes far away at certain distance, not only the RSSI can fluctuate, but also with increasing distance, the vibration frequency and 
amplitude will become bigger. Figure 2 gives the distribution of signal strength along with time when two nodes of transceiver stand away at distance of 1,2,3 meters; at each position, totally 105 groups of signals are observed; each group of signal at interval of $2 \mathrm{~s}$. As seen, RSSI value undulates around the mean value at fixed position. The longer the distance is, the fiercer the fluctuation becomes. As shown in Figure 2 and figure 1, the above mentioned model is in line with the actual situation.
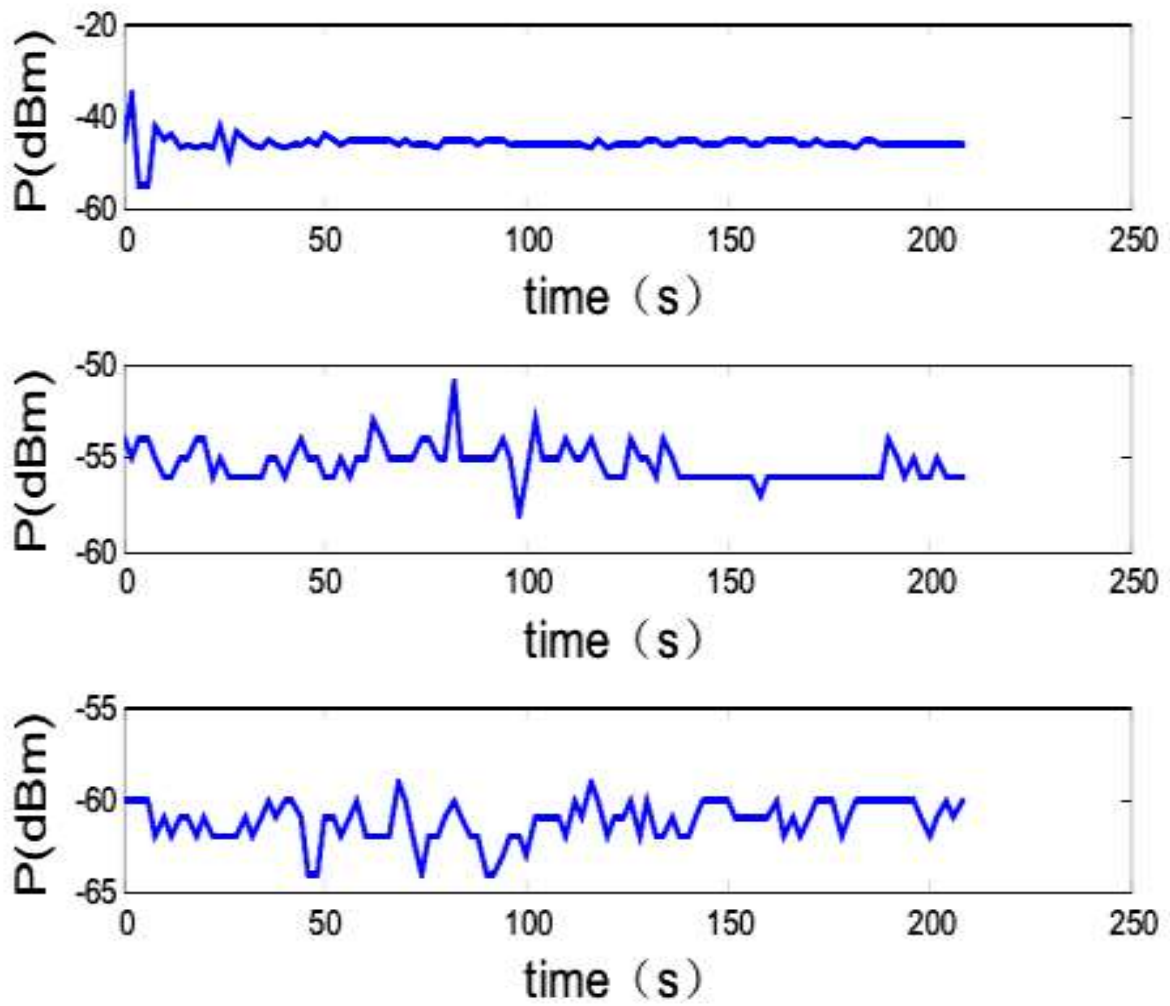

Figure 2. RSSI Fluctuations with time at the Distance of 1, 2, $3 \mathrm{~m}$ between Two Nodes

3.1.2. Experimental Result of SV Positioning based on RSSI: After RSSI signal transmit attenuation model is got, it's applied to evaluate the performance of distributed SUV positioning algorithm based on RSSI distance measurement. The test scenario is chosen at laboratory. In the $1 \mathrm{ab} 10 \times 10 \mathrm{~m}^{2}$, eight referential nodes are put around; also indoor there are devices and furniture. An unknown node sends signal then all referential nodes receive it; then report the RSSI value of measured signal to a gateway in the form of wireless signal, which is forwarded by gateway to PC server for positioning calculation.

Figure 3 shows the after 10 times calculated for average positioning results, which in the periphery of the square frame of eight red diamond for the reference node, within the 16 Blue solid round for the actual position of the unknown nodes, corresponding to 16 purple hollow circle based on the RSSI value is obtained by positioning the SV positioning results. The Euclidean distance between the actual position and the positioning position is used as the positioning error, the average location error of the 16 unknown nodes is $0.91 \mathrm{~m}$.

\subsection{Simulation Result}

In the experiment, very few sensor nodes are employed. In the limited lab space range, the effectiveness of the proposed method is tested. However, one of the most significant features of WSNs is big node scale, wide deployment space. In practical use, hundreds of 
thousands of sensor nodes are deployed. In environmental condition, we have no way to deploy such large-scale WSNs to test the performance of positioning algorithm. So here we take software simulation mode to assess quantitatively the effectiveness of the positioning algorithm, not only lots of sensor nodes being set and meeting requirements of wide application; and also the simulation can be implemented that factors affecting the positioning of WSNs exist in reality, such as measuring distance error, node communication distance.

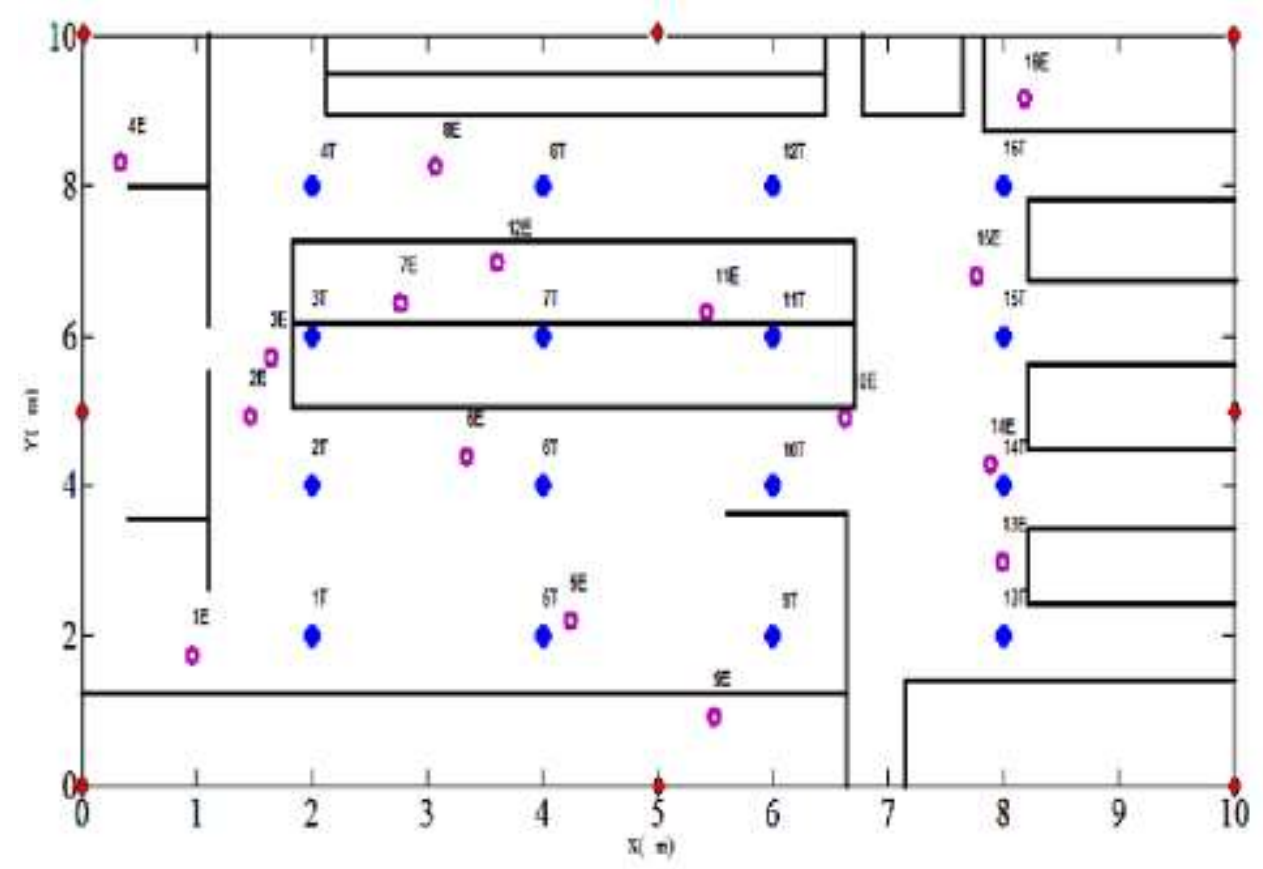

Figure 3. Experimental Localization Result

Choose Matlab as simulation platform to analyze and evaluate the performance of SV positioning algorithm. All methods are compiled with Matlab language, which are appraised from four aspects like: number of anchor node, communication range of sensor nodes, number of nodes and measuring distance error. For the first three elements, they can be adjusted through increasing node number and enhancing sensor node's communication range. With regards to measuring distance error, since there are a few distance ranging techniques and the measuring distance errors of different techniques differ largely, so in simulation experiment, the measuring distance between nodes can be obtained through Noisy Disk model:

$$
\mathcal{d}\left(x_{i}, x_{j}\right)=\left\{\begin{array}{l}
N\left(d\left(x_{i}, x_{j}\right), \sigma\right) \\
\text { Inf }
\end{array}\right.
$$

SV positioning algorithm can realize centralized and distributed positioning. In the below, we compare them.

At first, we do simulation evaluation of centralized SV positioning algorithm. About the arrangement of WSNs, there are regular deployment and random deployment. To assess the impact of anchor number on centralized positioning algorithm, Figure 4-5 presents positioning results of the two deployment methods, where red asterisk is position of anchor node; hollow circle is actual coordinate of unknown nodes; the straight line connected with circle refers to the distance between unknown node's actual position and estimated position. Two experiments both suppose all nodes can mutually communicate and measuring distance error is $15 \%$. 
In Figure 4, 81 unknown nodes are deployed in the area $100 \times 100 \mathrm{~m}^{2}$; when the number of anchor is respectively $4,7,10$ and 13, locate all unknown nodes then get the positioning error respectively $3.43 \mathrm{~m}, 3.71 \mathrm{~m}, 3.96 \mathrm{~m}$ and $3.42 \mathrm{~m}$.

In Figure 5, deploy randomly 80 nodes in the area $100 \times 100 \mathrm{~m}^{2}$; choose 4,6,8 and 10 nodes as anchor nodes; other nodes are used as unknown nodes to do positioning test; the positioning error is obtained separately $3.40 \mathrm{~m}, 2.96 \mathrm{~m}, 3.12 \mathrm{~m}$ and $3.39 \mathrm{~m}$. From two groups of experiments, we realize whether rule deployment or random deployment, the number of anchor nodes hardly has impact on positioning error.

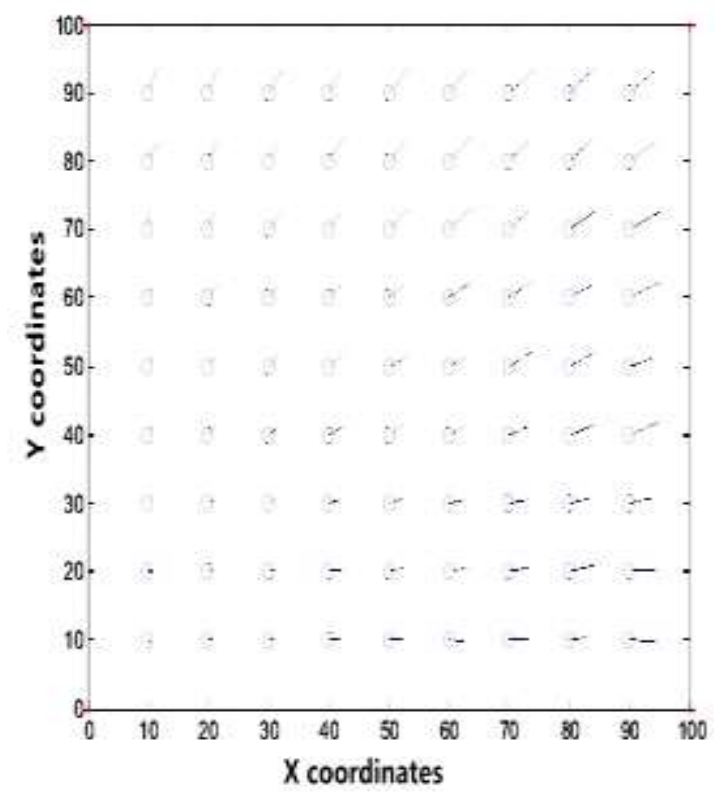

(a) Localization error $=3.43 \mathrm{~m}$

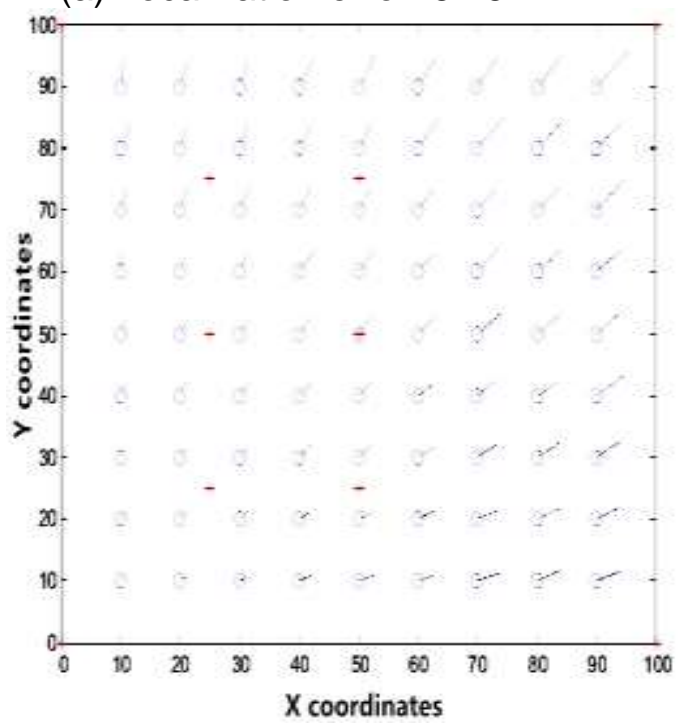

(c) Localization error $=3.96 \mathrm{~m}$

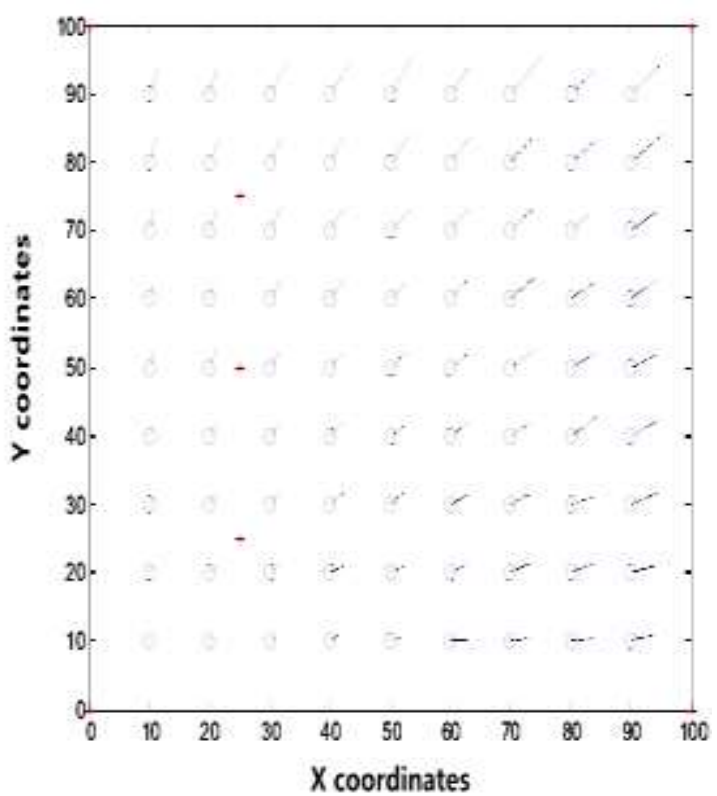

(b) Localization error $=3.71 \mathrm{~m}$

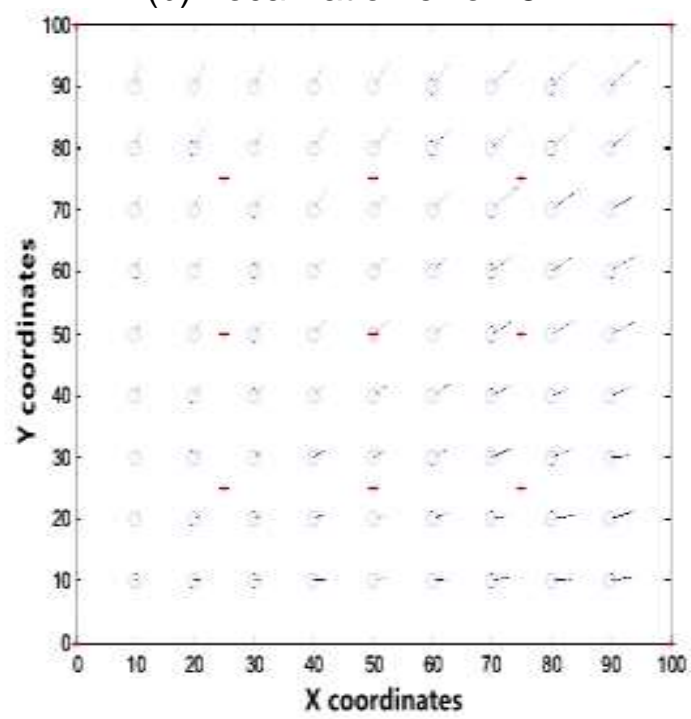

(d) Localization error $=3.42 \mathrm{~m}$

Figure 4. Localization Error Results of Regular Deployment. The Ranging Error is $15 \%$, and the Anchor Node Numbers are 4, 7, 10, 13, respectively 


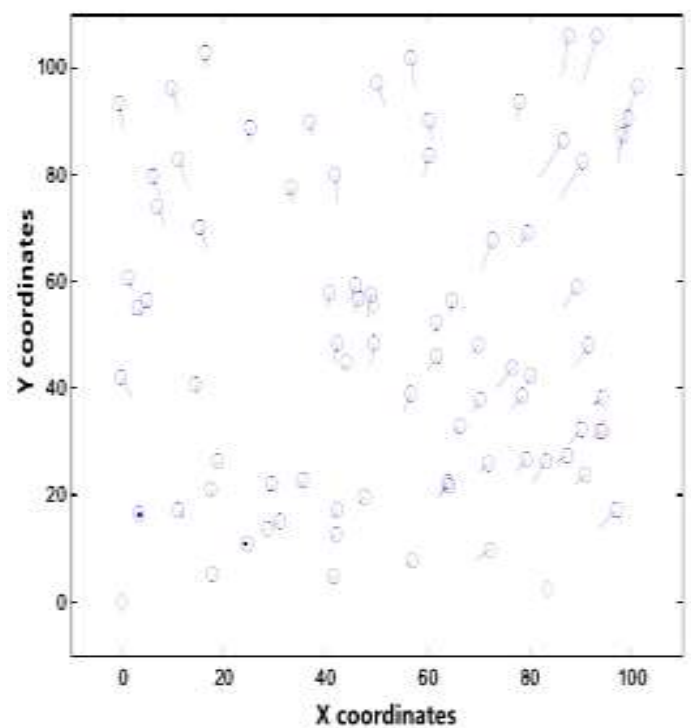

(a) Localization error $=3.40 \mathrm{~m}$

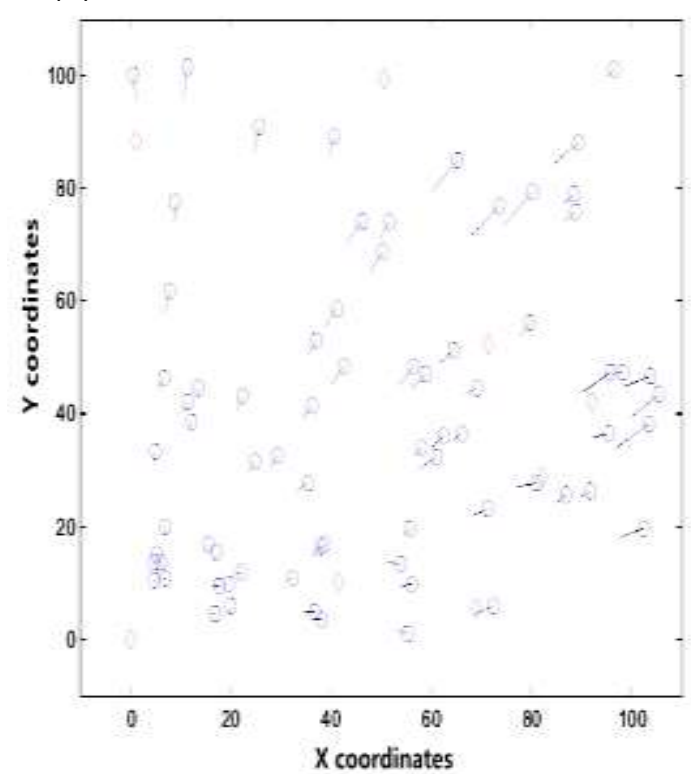

(c) Localization error $=3.12 \mathrm{~m}$

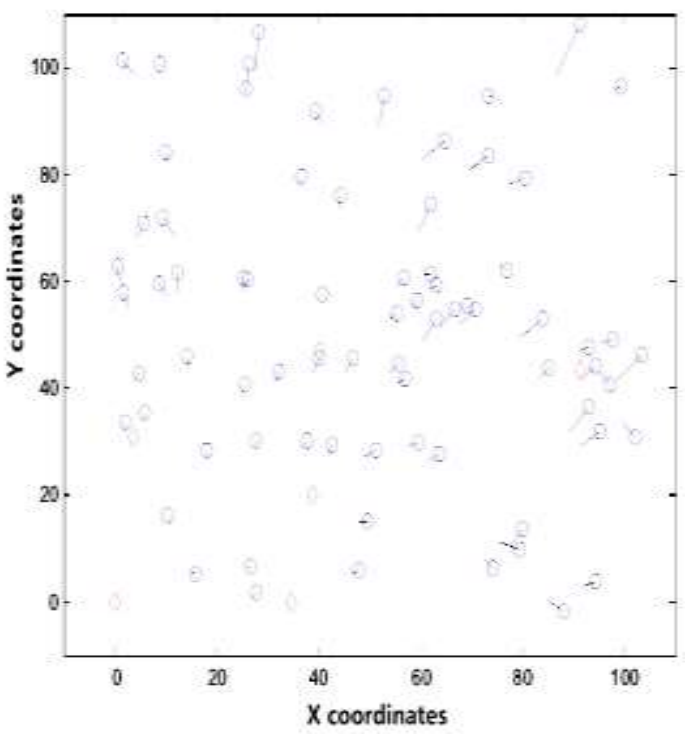

(b) Localization error $=2.96 \mathrm{~m}$

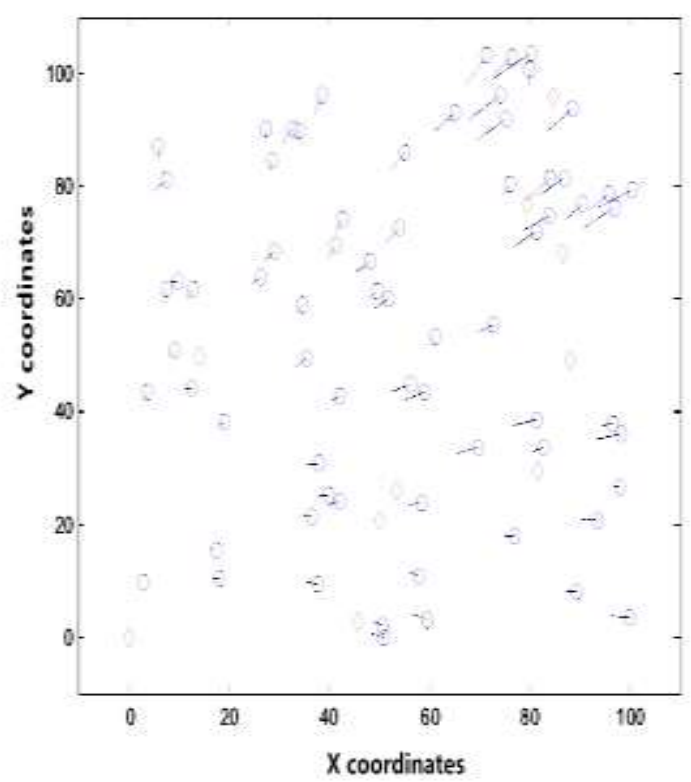

(d) Localization error $=3.39 \mathrm{~m}$

Figure 5. Localization Error Results of Random Deployment. The Ranging Error is $15 \%$, and the Anchor Node Numbers are 4, 6, 8, 10, respectively

We conduct second group of simulation test to find out the relationship between network connectivity and centralized SV positioning result. In the first group of experiment, suppose all nodes can communicate mutually. It should be noted that in practical use, it's not possible for all nodes to communicate mutually, especially when deploy area is big and/or node communication distance is small.

When direct communication between nodes can't be achieved, the shortest path between them is utilized to calculate the measuring distance between them. In order to solve the shortest path between two nodes, we use graph $\mathrm{G}$ to represent the entire network; sensor node in the network is vertex of graph G. Suppose the weighted matrix of graph G is $\mathrm{H}$. When there is one edge between vertex $X_{i}$ and $X_{j}$, i.e. $X_{i}$ and $X_{j}$ are in communication range. $H_{i j}=1$; otherwise, $H_{i j}=0$. That's because it's assumed all nodes communicate at the same distance. If node $X_{i}$ is not within $X_{j}$ 's communication range, 
node $X_{j}$ is not in $X_{i}$ 's communication range too. Therefore, weighted matrix $\mathrm{H}$ is a symmetric matrix; then we can get:

$$
H_{i j}=\left\{\begin{array}{l}
1, d\left(x_{i}, x_{j}\right) \leq C D \\
0, d\left(x_{i}, x_{j}\right)>C D
\end{array}\right.
$$

With weighted matrix and node measuring distance within communication range, we can define the shortest path of all nodes like:

$$
\mathscr{d}_{\text {min }}^{o}\left(x_{i}, x_{j}\right)=\min \left\{d\left(x_{i}, x_{j}\right) \times H_{i j},\left(d\left(x_{i}, x_{k}\right) \times H_{i j}+d\left(x_{k}, x_{j}\right) \times H_{i j}\right)\right\}
$$

To start, it tested in different communication ranges the node's network connectivity and relative positioning error results. In the area $100 \times 100 \mathrm{~m}^{2}, 70$ unknown nodes and 10 anchor nodes are arranged. Suppose the error of surveyed distance among all sensor nodes is $15 \%$ and all nodes' communication range is consistent. By changing node's communication distance, network connectivity can be adjusted. Node communication distance CD is made respectively $30 \mathrm{~m}, 40 \mathrm{~m}, 50 \mathrm{~m}$ and $60 \mathrm{~m}$; network connectivity is respectively $17.525,25.675,38.750,47.500$ and relevant positioning error is $9.02 \mathrm{~m}$, $5.72 \mathrm{~m}, 4.39 \mathrm{~m}$ and $2.93 \mathrm{~m}$. Figure 6 gives network connectivity and the positioning result under the above premises. With CD growing up, network connecting lines increase and node's positioning error bars become shorter.
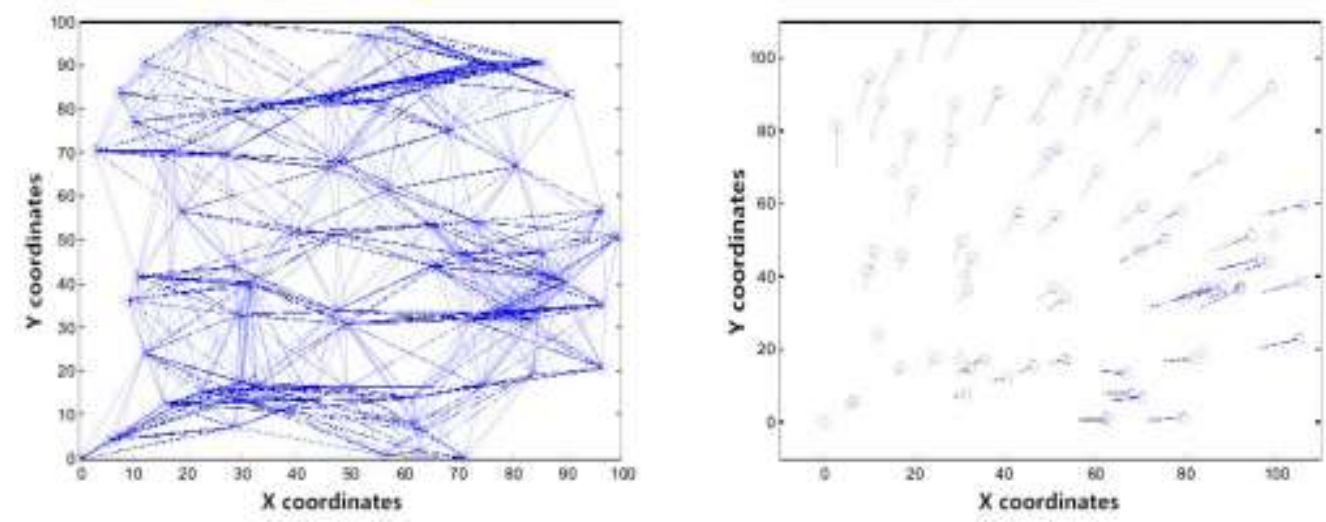

(a) $C D=30 \mathrm{~m}$
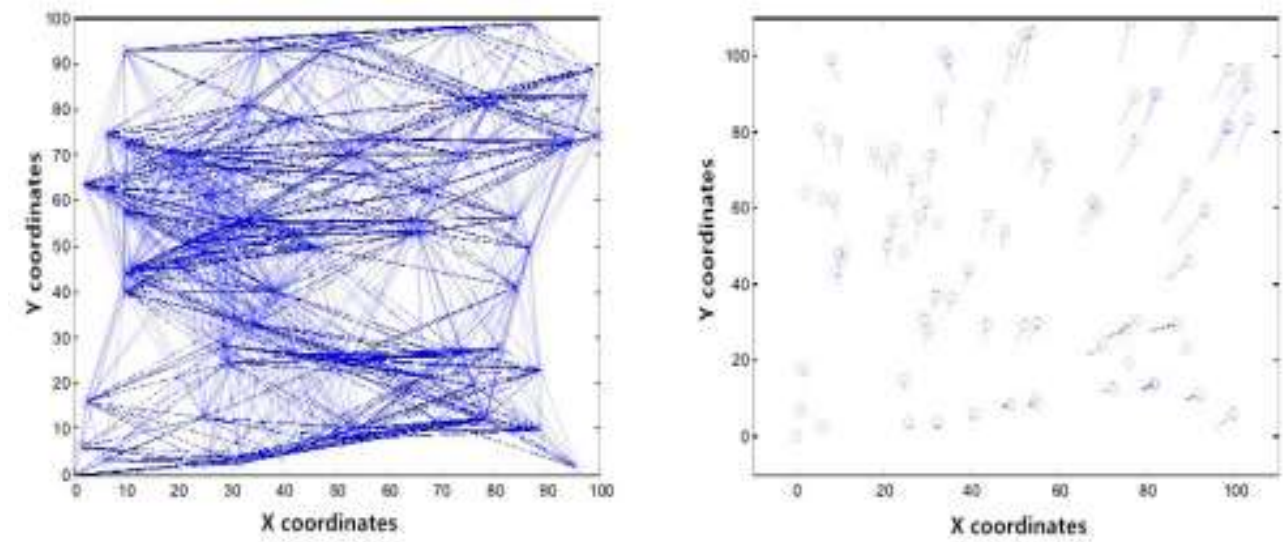

(b) $C D=40 \mathrm{~m}$ 

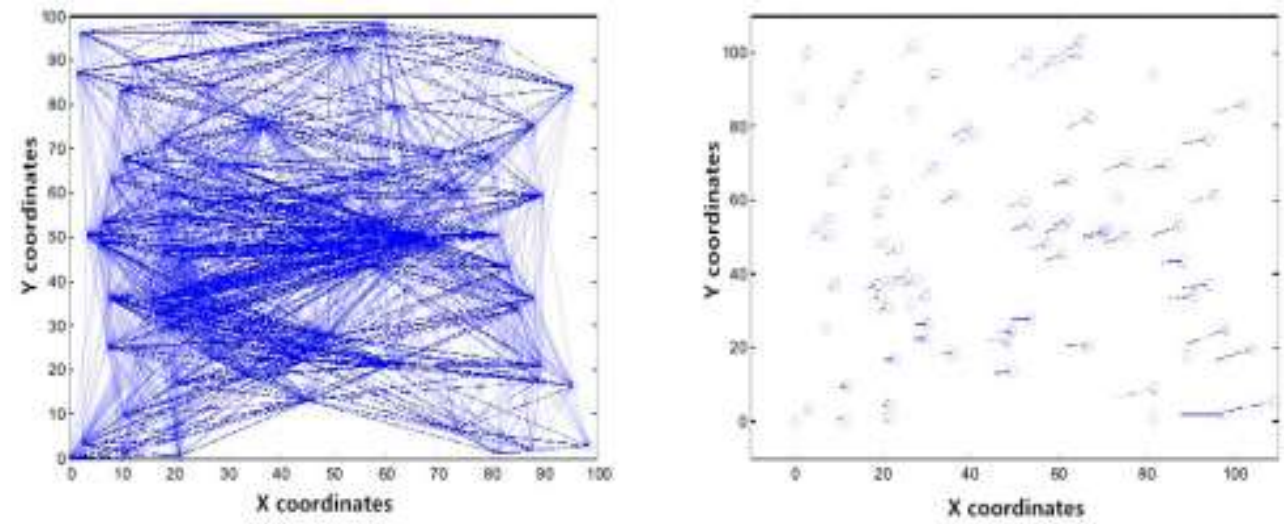

(c) $\mathrm{CD}=50 \mathrm{~m}$
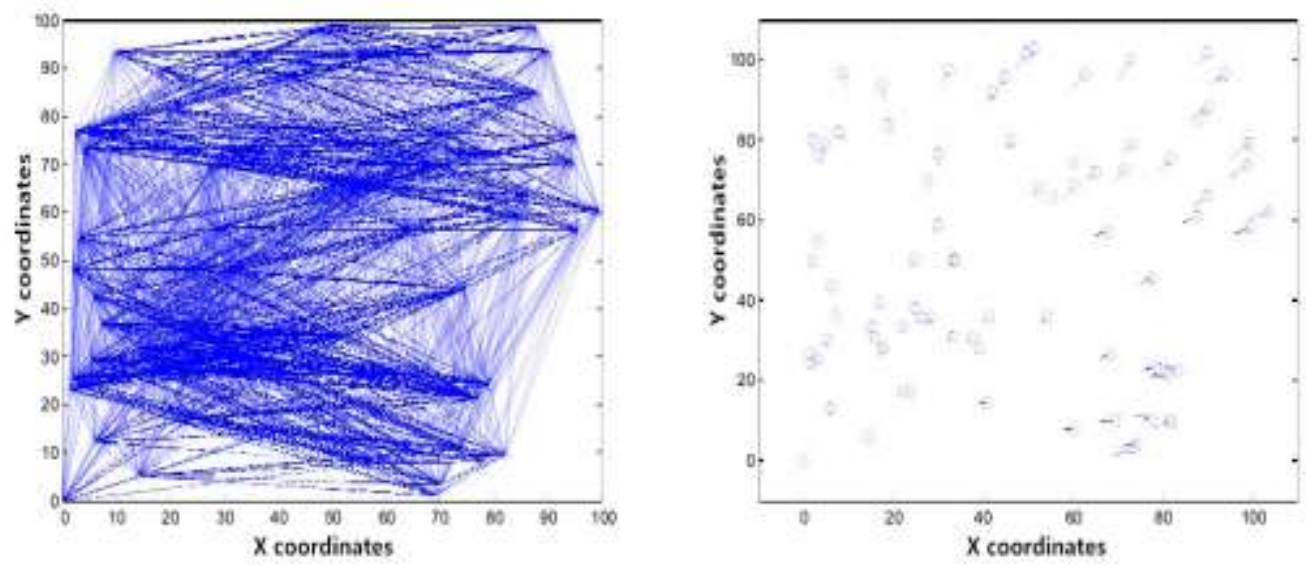

(d) $\mathrm{CD}=60 \mathrm{~m}$

\section{Figure 6. Network Connectivity (left) and Location Error Diagrams (right) at different $C D$}

In order to better evaluate the performance of the centralized SV location algorithm, the following were from the deployment of sensor nodes number $(\mathrm{NN})$, the ranging error between nodes $(\sigma)$, node communication distance (CD) positioning precision angle to view the algorithm, as an evaluation criterion for the performance of the proposed localization algorithm in the paper. Firstly, the effect of NN on the performance of SV location algorithm is evaluated.

Figure 7 (a) is in the case of $\sigma=0.01 \mathrm{a}=0.1$ ranging error, to add the sensor nodes to the network, the positioning error of the SV algorithm is obtained. It can be seen that when the $\mathrm{NN}$ is more than 40 , the position error of the three CD is decreased. When NN increases from 70 to 100 , the three curves become more and more smooth. In the case of $\mathrm{NN}=70, \mathrm{CD}=50$, the average positioning error is $3.85 \mathrm{~m}, \mathrm{NN}=100$, the mean position error is $3.23 \mathrm{~m}$, and $0.62 \mathrm{~m}$ is reduced.

Figure 7 (b) gives the sigma changes, the relationship between average positioning error and NN, similar to Figure 7 (a), the slope of the curve between NN 30 to 40 is greater than the slope of $\mathrm{NN}$ between 40 and 70, and the slope of the curve between NN 40 and 70 is larger than that between 70 and 100 . When is the $\mathrm{NN}>70$, the location error is small, which provides a reference for the deployment of the sensor network.

From 7 (b) can also be seen, influence of $\sigma$ on SV positioning is relatively large (spacing between the three curves obviously). In the case of $\mathrm{NN}=80, \sigma=0.1,0.2$ and 0.3 average positioning error values were $3.26 \mathrm{~m}, 6.11 \mathrm{~m}, 8.52 \mathrm{~m}$, the positioning accuracy decreased significantly. 

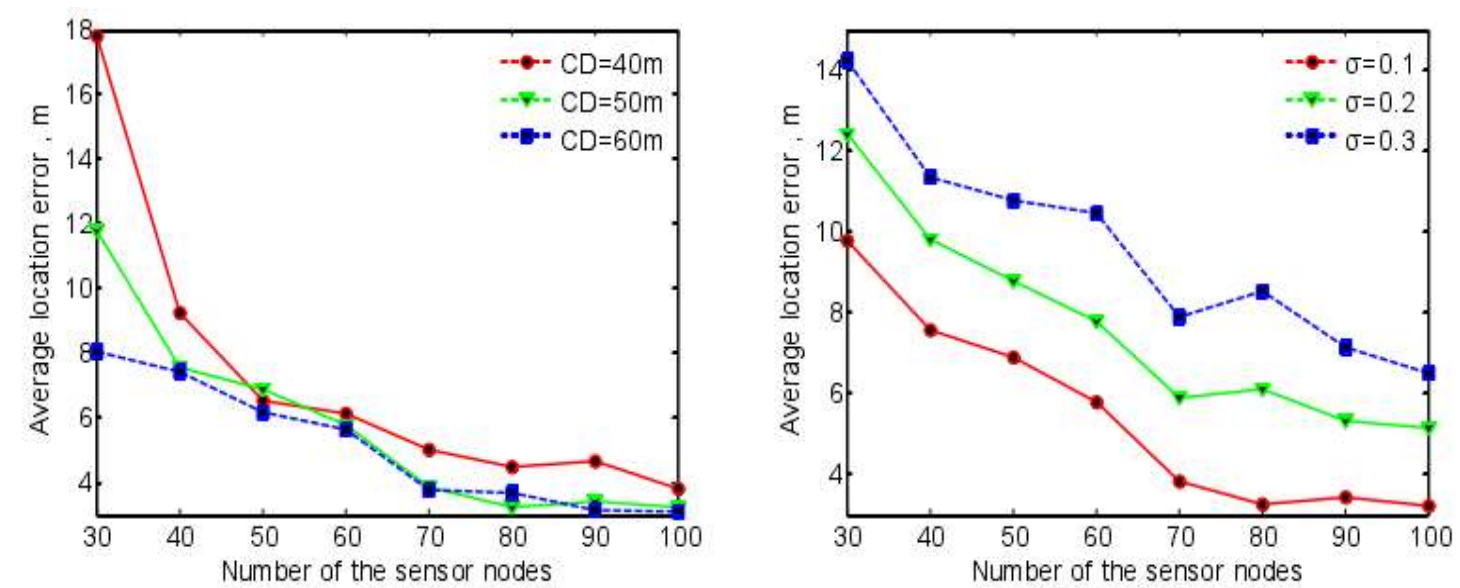

\section{Figure 7. Average Location Error as a Function of the Sensor Node Number at different Communication Distance (a) and different Ranging Error (b)}

Secondly, compare the effect of measurement error on the performance of a node localization algorithm. It is shown in Figure 8(a). The variation of NN for 70 and 100 curves was slower than that of $\mathrm{NN}$ for 40 . when $\sigma$ rises from $40 \%$ to $50 \%$, the positioning error was increased by $7.50 \mathrm{~m}, 6.63 \mathrm{~m}, 10.92 \mathrm{~m}$. It also verifies the relationship between the number of nodes and the location error in the upper group experiment.
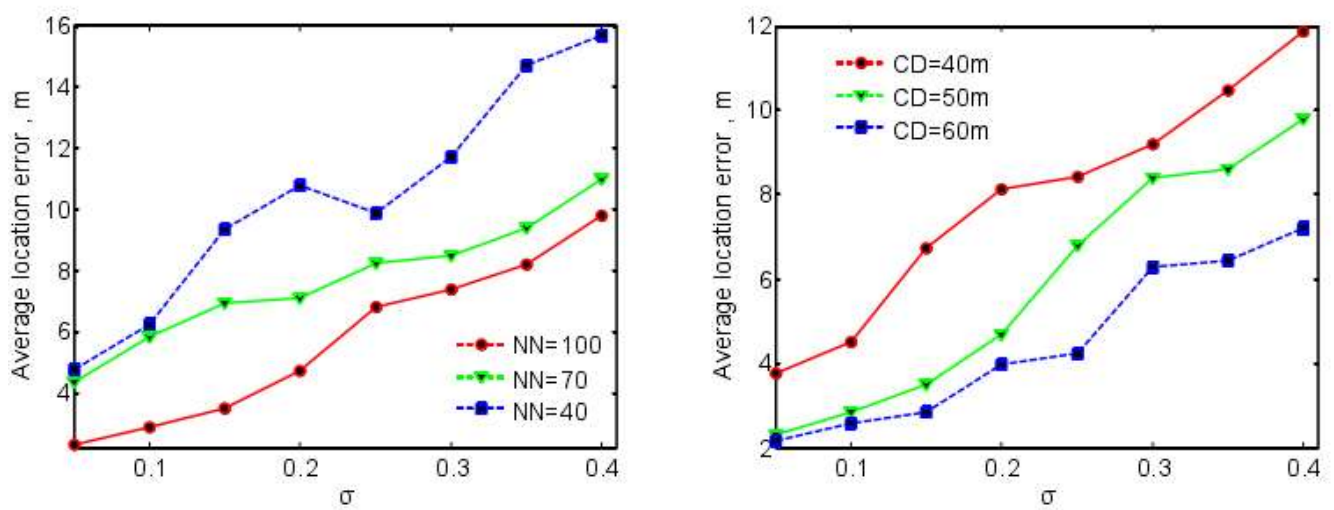

Figure 8. Average Location Error as a Function of the Ranging Error of Sensor Nodes at different Node Number (a) and different Communication Distance (b)

\section{Conclusion}

This paper proposed a new localization algorithm based on a set of statistical vectors. The algorithm is solved by simplifying the center coordinate matrix by the translation node coordinate system, directly to get the coordinates of the inner product matrix, using statistical vectors sets to construct the inner product matrix of coordinates, to realize the noise measurement of ranging noise, it can effectively reduce the positioning error caused by the ranging error. In this paper, we give the positioning algorithm based on statistical vector set of centralized localization, distributed localization scheme, and distributed localization algorithm is applied to the positioning experiment based on RSSI ranging technique. It is proved that the algorithm is effective. 


\section{Acknowledgement}

This work was supported by design and implementation of teaching call center system based on CTI integration technology (XJK013CXX016), the National Natural Science Foundation of China (61571188) and the excellent youth research project of The Education Department of Hunan Province (No.15B125)

\section{References}

[1] W. Xiaoping, L. Jun and S. Changxiang, "Localization theory and algorithm of wireless sensor networks", computer research and development, vol. 3, (2011), pp. 353-363.

[2] P. Yu and W. Dan, "A survey of wireless sensor network localization technology", Journal of electronic measurement and instrument, vol. 5, (2011), pp. 389-399.

[3] H. Yanli, "Centroid localization algorithm for wireless sensor networks", computer simulation, vol. 5, (2011), pp. 163-166.

[4] C. Chi, "Localization algorithm and data gathering protocol for wireless sensor networks with mobile nodes", Central South University, (2012).

[5] S. Shunyuan, "Localization algorithm and Application Research of wireless sensor networks", Jiangnan University, (2014).

[6] G. Sheng, "Difficult based on the improved RSSI wireless sensor network node localization algorithm", Application Research of computers, vol. 5, (2012), pp. 1867-1869.

[7] S. B. Cai, H. Zhenguo and H W. Pan, "jade like stone, With penalty function of wireless sensor network particle group positioning algorithm”, Journal of computer research and development, vol. 6, (2012), pp. 1228-1234.

[8] P. Wenjing, "Localization algorithm in wireless sensor network research", Jilin University, (2013).

[9] L. Jing, "Wireless sensor network node positioning algorithm”, Dalian Maritime University, (2013).

[10] C. Jing, "Research on Localization Algorithm of wireless sensor network for forest fire monitoring", Beijing University of Posts and Telecommunications, (2015).

[11] S. Hao, "Localization algorithm of wireless sensor networks in complex environment", Zhejiang University of Technology, (2015).

[12] M. S. Alejandro and E. L. Esteban, "An Accurate Radio Channel Model for Wireless Sensor Networks Simulation", Journal of Communications and Networks, vol. 7, no. 4, (2005), pp. 1-7.

[13] X. Li, "Performance study of RSS-Based Location Estimation Techniques for Wireless Networks", Proceedings IEEE Military Communication, Conference, Atlantic NJ, vol. 2, (2005), pp. 1064-1068

[14] C. Z. Sahinoglu, "The Cramer-Rao Bounds of hybrid TOA/RSS and TDOA/RSS Location Estimation Schemes", IEEE Communication Letter, vol. 8, no. 4, (2004), pp. 626-628.

\section{Authors}

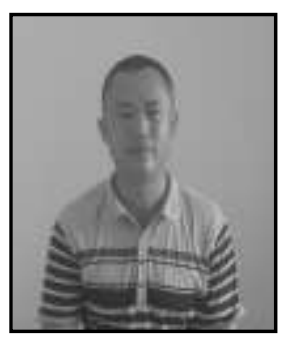

Xuelong Zhang, he received his M.S degree from Hunan Normal University. $\mathrm{He}$ is an associate professor in Changsha Normal University. His research interests include Circuits and systems.

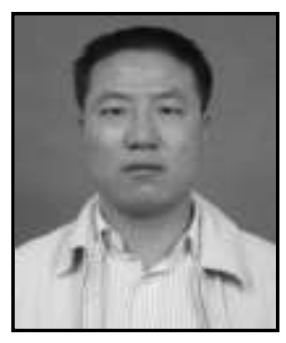

Hao Liu, he received his bachelor degree from National University of Defense Technology, China, in 2002, received his master degree in Huazhong University of Science \& Technology, China, in 2007, and then received his doctor degree in South China University of Technology, China, in 2010. Currently, he is an associate professor of Hunan University of Humanities, Science and Technology, China. His research interests include P2P network and network security. 
International Journal of Future Generation Communication and Networking Vol. 10, No. 1 (2017) 